\title{
Wirklichkeitserzählungen. Felder, Formen und Funktionen nicht-literarischen Erzählens
}

\author{
Christian Klein/Matias Martínez
}

Erzählen ist eine grundlegende Form unseres Zugriffs auf Wirklichkeit. In den verschiedensten Bereichen der alltäglichen Lebenswelt und nicht zuletzt auf den Gebieten wissenschaftlicher Erkenntnis orientieren und verständigen wir uns mit Hilfe von Erzählungen. Reportagen des investigativen Journalismus, Selbstdarstellungen von Politikern im Wahlkampf, Erlebnisberichte in Internetblogs, Anamnesen im medizinischen Patientengespräch, Plädoyers vor Gericht, Vermittlungen von Verhaltensnormen in populärer Ratgeberliteratur, Heilserzählungen im Gottesdienst, Fallgeschichten in juristischen Lehrbüchern, ökonomische Prognosen von Kursverläufen - all diese Kommunikationen erfolgen wesentlich in erzählender Form. Anders als in den erfundenen Geschichten der Literatur bezieht man sich in diesen Erzählungen direkt auf unsere konkrete Wirklichkeit und trifft Aussagen mit einem spezifischen Geltungsanspruch: 'So ist es (gewesen)، ${ }^{1}$ Solche Erzählungen mit unmittelbarem Bezug auf die konkrete außersprachliche Realität nennen wir IVirklichkeitserväblungen.

In den vergangenen Jahrzehnten wurde die referentielle Leistung sprachlicher Kommunikation im Zeichen strukturalistischer und poststrukturalistischer Theorien allzu oft zugunsten eines pauschalen Panfiktionalismus< unterschlagen. ${ }^{2}$ Zweifellos konstruieren Wirklichkeitserzählungen in erheblichem Maße eine Realität; aber sie sind eben auch auf eine intersubjektiv gegebene Wirklichkeit bezogen. Wirklichkeitserzählungen sind sowohl konstruktiv als auch referentiell darin liegt ihre besondere erkenntnistheoretische Bedeutung. Es gilt, den referentiellen Aspekt von Wirklichkeitserzählungen angemessen zu berücksichtigen, ohne deren konstruktive Elemente zu vernachlässigen.

\section{Literarisch-fiktionales und faktuales Erzählen}

Was unterscheidet Wirklichkeitserzählungen von literarischen Erzählungen? Wir wollen den Unterschied zwischen diesen beiden Arten des Erzählens präziser als den Gegensatz zwischen faktualen und fiktionalen Texten bestimmen. Häufig begegnet man der Ansicht, das Besondere an literarischen Erzählungen (an Romanen, Kurzgeschichten etc.) sei, dass sie keine realen, sondern fiktive (d.h. erfundene) Geschehnisse schilderten. In der bekannten Unterscheidung der aristoteli-

1 Wir werden unten zeigen, dass sich der faktuale Wirklichkeitsbczug auch in anderer Weise ausdrücken kann: sso soll es sein oder sso wird es seink.

2 Vgl. zur >Panfiktionalismuse-Debatte z.B.: Peter Blume: Fik.tion und W'eltwissen. Der Beitrag nichtfiktionaler Konzepte ₹ur Sinnkonstitution fiktionaler Erzäbliteratur, Berlin 2004, S. 12-16. 
schen Poetik. (1451b) ausgedrückt: Der Dichter teilt mit, was geschehen könnte, während der Geschichtsschreiber (als Repräsentant nicht-literarischen Erzählens) das tatsächlich Geschehene darstellt. Die Frage danach, ob ein geschildertes Geschehen fiktiv ist oder nicht, bezieht sich darauf, was erzählt wird. Entscheidend für die Bestimmung eines dargestellten Geschehens als real oder fiktiv ist die Referenz, nämlich die Frage, ob der im Text dargestellte Sachverhalt in der auBersprachlichen Realität tatsächlich der Fall war/ist oder nicht. ${ }^{3}$

Mit dieser Unterscheidung wird allerdings der besondere Charakter fiktionalliterarischer Erzählungen nicht angemessen erfasst. Während ‘fiktiv im Gegensatz zu reak steht und die Frage nach der Fiktivität auf den ontologischen Status der dargestellten Sachverhalte zielt, steht sfiktionak im Gegensatz zu ifaktuak und bezeichnet einen bestimmten Modus von erzählender Rede. ${ }^{4}$ Faktuale Erzählungen (z.B. Thomas Nipperdeys Deutsche Geschichte 1800-1918) sind Teil einer realen Kommunikation und bestehen aus Sätzen, die vom Leser als wahrheitsheischende Behauptungen des Autors (Thomas Nipperdey) verstanden werden. Fiktionale Texte (z.B. Günter Grass' Roman Die Blechtrommel) sind ebenfalls Teil einer realen Kommunikation, in der ein realer Autor (Günter Grass) Sätze produziert, die von einem realen Leser gelesen werden. Fiktionale Texte sind jedoch komplexer als faktuale, weil sie außer der realen auch noch eine zweite, imaginäre Kommunikationssituation gestalten. In dieser zweiten Situation kommt ein erfundener Erzähler zu Wort (z.B. der fiktive Ich-Erzähler Oskar Matzerath). Die fiktionale Erzählung enthält also sowohl eine reale wie eine imaginäre Kommunikation und stellt insofern eine skommunizierte Kommunikation" dar. ${ }^{5}$ Anders als der reale Sprecher einer faktualen Rede ist das fiktive Aussagesubjekt der fiktionalen Rede nicht an die nnatürlichen< Beschränkungen menschlicher Rede gebunden und kann deshalb z.B. ungestraft die Position eines allwissenden Erzählers einnehmen. Und der reale Autor eines fiktionalen Textes kann nicht für den Wahrheitsgehalt der in seinem Text aufgestellten Aussagen verantwortlich gemacht werden, weil er diese zwar produziert, aber nicht behauptet - vielmehr ist es der imaginäre Erzähler, der diese Sätze mit Wahrheitsanspruch behauptet. Die reale Kommunikation zwischen Autor und Leser findet hier nur indirekt statt und ähnelt dem Zitieren der Rede eines anderen. Denn auch beim Zitieren übermittelt man Sätze, die jemand anders behauptet hat, die aber nun, im Akt des Zitierens, ohne behauptende Kraft weitergegeben werden. Fiktionale Rede stellt Sachverhalte als wirkliche dar, ohne jedoch

3 Wie es sich wiederum mit dieser Realität< verhält, inwiefern sie ein Konstrukt unseres Erkenntnisapparats ist, ob sie absolut gegeben oder kulturell relativ ist - das sind fundamentale erkenntnistheoretische Fragen, die nicht in unseren Zusammenhang gehören. Entscheidend für Wirklichkcitserzählungen ist der mit ihnen verbundene - und sie von fiktionalen Exzählungen unterschcidende - Geltungsanspruch, reale Sachverhalte darzustellen.

4 Der Terminus sfaktuale Erzählung، (bəw. récit factuek) wurde cingeführt von Gérard Genette: Fiktion und Diktion, München 1992, S. 11-40. Zur Unterschcidung von sfiktional und sfiktiv vgl. Frank Zipfel: Fiktion, Fiktivität, Fiktionalität. Analysen zur Fiktion in der Literatur und zun Fiktionsbegriff in der Literaturwissenschaft, Berlin 2001, insb. S. 61-68; Matías Martínce/Michael Scheffel: Einfihbrung in die Erzäbltheorie, 7. Aufl., München 2007, S. 9-20.

5 Dieter Janik: Die Kommunikationsstruktur des Erzäblwerks. Ein semiologisches Modell, Bebonhau$\operatorname{sen} 1973$, S. 12 
eine Referenz dieser Darstellung auf unsere Wirklichkeit zu behaupten. Mit anderen Worten: Unter allen möglichen Verfassern lügen die Dichter am wenigsten, weil sie - im Gegensatz z.B. zu den Geschichtsschreibern - in ihren Werken gar nichts selbst behaupten, sondern einen imaginären Erzähler erfinden, der immer nur etwas mit Bezug auf seine fiktive Welt behauptet. Dichter erzählen von etwas, das nicht ist, und so ist es sinnlos, sie des Irrtums, der Lüge oder Täuschung überführen zu wollen.

Die Rezeption faktualer Erzählungen geht indes mit einer anderen Leseerwartung einher: Thr Leser erwartet nicht die Schilderung eines möglichen (oder gar fantastisch-unmöglichen), sondern eines wirklichen Geschehens. Textpragmatisch zeichnen sich faktuale Erzählungen im Gegensatz zu fiktionalen dadurch aus, dass der Autor zugleich auch der Erzähler seines Textes ist. Er muss für die Wahrheit der vorgebrachten Behauptungen einstehen. Verfasser faktualer Texte schließen mit ihren Lesern eine Art Abkommen. Indem sie ihren Text als faktual markieren, sichern sie zu, dass sie wahrhaftig, knapp, klar und relevant berichten. Gérard Genette spricht in diesem Zusammenhang von einer "Wahtheitsverpflichtung « des Autors faktualer Texte, Philippe Lejeune von einem »Pakt« ${ }^{6}$

Woran erkennt man aber, ob man einen fiktionalen oder einen faktualen Erzähltext vor sich hat? Die Fiktionalität eines Textes kann, erstens, textpragmatisch dadurch angezeigt werden, dass Name und Person des realen Autors ( $z . \mathrm{B}$. Günter Grass) nicht mit dem übereinstimmen, was aus dem Text über den Erzähler (z.B. den »Insassen einer Heil- und Pflegeanstalt« Oskar Matzerath) zu erschließen ist. Zweitens können paratextuelle Informationen wie Gattungsbezeichnungen (»Roman«) oder andere Hinweise (»Personen und Handlung des $\mathrm{Bu}-$ ches sind frei erfunden () einen Text wie Die Blechtrommel von vornherein als fiktional kennzeichnen. Drittens sind für fiktionales Erzählen bestimmte textinterne Merkmale charakteristisch, die eine übermenschliche Allwissenheit des Erzählers voraussetzen; dazu gehören insbesondere Einblicke des Erzählers in die Gedanken- und Gefühlswelt seiner Figuren. ${ }^{7}$ Allerdings ermöglichen diese textinternen Signale keine trennscharfe Abgrenzung zwischen fiktionalen und faktualen Texten. Nicht alle fiktionalen Texte enthalten Charakteristika allwissenden Erzählens, weshalb diese Kennzeichen nicht als ein notwendiges Kriterium für Fiktionalität gelten können. Und andererseits greifen auch faktuale Texte, beispielsweise des Journalismus oder der Geschichtsschreibung, gelegentlich zu Darstellungsmitteln, die streng genommen den Standpunkt eines allwissenden Erzählers voraussetzen (z.B. die wörtliche Wiedergabe unprotokollierter Dialoge oder Aussagen über Gedanken und Gefühle historischer Personen), ohne jedoch deswegen ihren faktualen Geltungsanspruch aufzugeben; allerdings muss der Autor hier seine fiktionalisierenden Erzählverfahren durch den Verweis auf eigene Recherchen, Dokumente o.ä. als plausible Vermutungen faktual legitimieren. Folglich können solche textinternen Merkmale auch kein hinreichendes Kriterium für die Entschei- 
dung sein, ob nun ein fiktionaler oder faktualer Erzähltext vorliegt - sie liefern allenfalls Hinweise und Signale. Die Klassifikation eines Textes als fiktional oder faktual ist eine Entscheidung, die letztlich auf textpragmatischer Ebene getroffen wird. ${ }^{8}$

Durch die Tatsache, dass auch in (manchen) faktualen Texten Erzähltechniken Verwendung finden, die gemeinhin fiktionalem Erzählen vorbehalten zu sein scheinen, sehen Vertreter eines $>$ Panfiktionalismus ihre radikal-konstruktivistische Auffassung gestützt, derzufolge jede sprachliche Darstellung von Wirklichkeit sich konstruktiver Fiktionen bedienen müsse, eine Trennung zwischen fiktionaler und nicht-fiktionaler Rede mithin unmöglich sei. Doch wird hier das Kind mit dem Bade ausgeschüttet. Ergiebiger ist es, die Verwendung genuin fiktionaler Erzählformen in faktualen Texten ernst zu nehmen, ohne deshalb gleich den Referentialitätsanspruch dieser Texte abzustreiten, der sie fundamental von den Geschichten der fiktionalen Literatur unterscheidet.

Der grundsätzliche Unterschied zwischen fiktionalen und faktualen Erzählungen wird auch nicht durch die Existenz von `Borderline-Texten aufgehoben, die auf unterschiedliche Art und Weise mit dieser Grenze spielen. Solche Fälle machen allerdings in besonderem Maße darauf aufmerksam, dass die Opposition fiktional vs. faktual nicht trennscharf ist, sondern verschiedene Kombinationen und Hybridisierungen erlaubt. Unterscheidet man zwischen der Fiktivität der erzählten Geschichte (besitzen die erzählten Sachverhalte eine Referenz in unserer Wirklichkeit oder nicht?) und der Fiktionalität der Erzählrede (wahrheitsheischende Rede des realen Autors oder imaginäre Rede eines fiktiven Erzählers?), lassen sich verschiedenartige Grenzfälle unterscheiden.

\section{Faktuale Erzäblungen mit fiktionalisierenden Erzäblverfabren}

$\mathrm{Zu}$ diesem Typ gehören z.B. Texte des New Journalism wie Truman Capotes $I n$ Cold Blood (1965). Capote erzählt hier die authentische Geschichte der Ermordung einer Familie durch zwei Jugendliche und verwendet dabei literarischer Erzähltechniken wie erlebte Rede oder die Wiedergabe von (nicht durch Protokolle o.ä. dokumentierten) Gesprächen in wörtlicher Rede, also Verfahren, die, streng genommen, einen allwissenden Erzähler voraussetzen. Capotes Anspruch, eine wahre Geschichte zu erzählen, wird durch diese fiktionalisierenden Elemente aber nicht außer Kraft gesetzt.

8 V'gl. Irmgard Nickel-Bacon/Norbert Groeben/Margit Schreicr: "Fiktionssignale pragmatisch. Fïin medienübergcifendes Modell zur Unterscheidung von Fiktion(en) und Realität(en)«, Poetica 32 (2000), Heft 3/4, S. 267-299, und Meike Herrmann: Fiktionalität gegen den Strich lesen. Was kann die Fiktionstheorie z"n einer Poetik des Sachbuchs beitragen? Reihe Arbeitsblätter für die Sachbuchforsclsung, Nr. 7. (als Download unter http://www.sachbuch forschung.de/html/literatur.]rtml, Aufruf 15.5.09). Umfangreichere kontrastive Untersuchungen über die Unterschiede zwischen fiktionalem und nicht-fiktionalem Erzählen aus pragmatischer Perspektive stehen noch aus. 
Faktuale Erä̈blungen mit fik:tiven Inbalten

$\mathrm{Zu}$ diesem Typ gehören Texte, die im faktualen Modus auftreten und einen referentiellen Geltungsanspruch erheben, aber gleichwohl Unwahres erzählen. Innerhalb dieser Gruppe wäre zu unterscheiden zwischen Texten, die (a) irrtümlich Unwahres behaupten und solchen, die es (b) wider besseres Wissen tun. Eine Biographie, die etwa guten Glaubens aus Stasi-Unterlagen, welche sich dann im Nachhinein als Erfindungen eines IM herausstellen, einen Lebenslauf rekonstruiert, würde zur ersten Gruppe gehören, die von Konrad Kujau gefälschten Tagebücher Adolf Hitlers zählen zur zweiten. Solche Texte erheben zwar den Anspruch, auf reale Tatsachen (die vermeintlich protokollierten Lebensgewohnheiten des Bespitzelten, Hitlers Alltag) zu referieren, lösen diesen Referenzanspruch jedoch nicht ein. Aber die mangelnde Referenz macht sie nicht schon zu fiktionalen Texten, sondern zu defizitären (nämlich falschen oder lügnerischen) faktualen Texten. Ausschlaggebend für die Unterscheidung zwischen fiktionalen und faktualen Texten ist der jeweils mit den Texten erhobene Geltungsanspruch.

\section{Fik:tionale Emähblungen mit faktualen Inbalten}

Selbstverständlich werden in vielen fiktional-literarischen Erzählungen Personen, Orte oder Ereignisse aus unserer Wirklichkeit erwähnt (z.B. Adolf Hitler, Danzig und der Zweite Weltkrieg in der Blechtrommel). In fiktionaler Rede kommt solchen Elementen jedoch ein grundsätzlich anderer Status als in Wirklichkeitserzählungen zu. ${ }^{9}$ Und sie geben zumeist nur den Horizont für eine ansonsten fiktive Geschichte $\mathrm{ab}$, die vielleicht in unserer Wirklichkeit möglich sein mag, sich aber eben nicht so zugetragen hat. Auch innerhalb dieser Gruppe gibt es sicherlich ein breites Spektrum an Varianten. So unterliegen historische Romane strengeren Erwartungen an historische Triftigkeit als kontrafaktische Romane wie Robert Harris' Fatberland (1992), der eine Geschichte aus Berlin im Jahr 1964 erzählt - in einer Welt, in der Deutschland und seine Verbündeten den Zweiten Weltkrieg gewonnen haben.

\section{Fiktionale Emääblungen mit faktualem Redenodus}

Manche literarische Werke vermeiden Erzählverfahren und Inhalte, die sie sofort als fiktional erkennbar machen, und inszenieren sich spielerisch als faktual. Auch hier findet sich ein breites Spektrum von Möglichkeiten. Einerseits gibt es Romane mit faktualisierenden Erzählverfahren wie Herausgeberberichten, Quellenangaben o.ä., wie etwa in Miguel de Cervantes' Don Quijote (1605/15), die unschwer als literarische Konventionen $\mathrm{zu}$ erkennen sind. Andererseits gibt es Texte, die alles daran setzen, ihren fiktionalen Status zu kaschieren, wie etwa Wolfgang Hildesheimers Text Marbot. Eine Biographie (1981) über den fiktiven englischen Kunsttheoretiker Andrew Marbot.

9 Vgl. hierzu Blume (Anm. 2). 


\section{Wirklichkeitserzählungen}

Dreh- und Angelpunkt für die Bestimmung jener Art von Texten, um die es in diesem Band geht, ist ihr referentieller Anspruch - daher unsere Begriffsschöpfung Wirklichkeitserquählungen: Wesentliches Merkmal dieser Erzählungen ist ihr konkreter Bezug auf reale Begebenheiten, auf Wirklichkeit: Sie liefern Aussagen über konkrete Sachverhalte unserer Lebenswelt. Freilich ist nicht jede sprachliche Kommunikation mit Referenz auf Wirklichkeit gleich eine Wirklichkeitserzäblung - unter einer Erzählung verstehen wir die sprachliche Darstellung eines Geschehens, also einer zeitlich organisierten Abfolge von Ereignissen. Der Begriff Wirklichkeitserzählung weist außerdem darauf hin, dass Gegenstand dieses Bandes mündliche oder schriftliche Erzählungen sind, die nicht literarisch in einem engeren Verständnis sind, weil sie eben (a) einen Anspruch auf unmittelbare Verankerbarkeit in der außersprachlichen Wirklichkeit erheben, sich also im Zweifelsfall auf reale Sachverhalte oder Begebenheiten beziehen, und/oder (b) keinen hohen Grad an Poetizität aufweisen.

Auf der Grundlage dieser Überlegungen lassen sich nun einige Merkmale jener Art von Erzählungen benennen, die wir als Wirklichkeitserzählungen bezeichnen. Wirklichkeitserzählungen beanspruchen, auf reale, räumlich und zeitlich konkrete Sachverhalte und Ereignisse zu referieren und sind in diesem Sinne faktuale Erzählungen. Im Rahmen ihres faktualen Geltungsanspruchs lassen sich drei Varianten von Wirklichkeitserzählungen unterscheiden. Mit Wirklichkeitserzählungen ist der Anspruch verbunden, dass die dargestellten Ereignisse entweder (a) tatsächlich stattgefunden haben oder dass sie (b) stattfinden sollten oder dass sie (c) stattfinden werden. Dementsprechend lassen sich drei Typen von Wirklichkeitserzählungen bestimmen:

(a) Deskriptive Wirklichkeitserzäblungen: Die Funktion dieses Typs ist die Darstellung realer Sachverhalte. Der hier erhobene Geltungsanspruch orientiert sich an der Dichotomie wahr vs. falsch. Als Beispiele wären die Rekonstruktionen von Ereignissen in der Geschichtsschreibung oder im Journalismus anzuführen.

(b) Nomative Wirklichkeitserväblungen: Bei diesem Typ wird ein enwünschter $\mathrm{Zu}$ stand von Wirklichkeit geschildert mit dem Ziel, eine bestimmte (gesellschaftliche oder individuelle) Praxis zu regulieren, das geschieht durch exemplifikatorische Darstellungen (menschlicher Handlungen). Der Geltungsanspruch orientiert sich an der Dichotomie sichtig handeln vs. falsch handelnk. Beispiele wären Verhaltens-Ratgeber, moralische Handlungsnormen oder juristische Gesetze.

(c) Voraussagende Wirklichkeitseräählungen: Bei diesem Typ wird ein erwarteter künftiger Zustand der Wirklichkeit geschildert. Seine Funktion ist die Festlegung allgemeiner Strukturmerkmale. Der erhobene Geltungsanspruch orientiert sich an der Dichotomie splausibel vs. unplausibek. Als Beispiele wären hier naturwissenschaftlich begründete Voraussagen (etwa über den globalen Klimawandel), medizinische Prognosen (etwa über das Herzinfarktrisiko ei- 
nes Patienten) oder Modellierungen künftiger Aktienverläufe im wirtschaftlichen Diskurs zu nennen.

Die Unterteilung ist idealtypisch - Wirklichkeitserzählungen kombinieren häufig diese Typen. Überhaupt sind Wirklichkeitserzählungen häufig hybride Texte, wobei sich diese Hybridität auf zwei Ebenen abspielen kann: (1) Auf der Ebene der histoire (der Ebene des Erzählten) werden mitunter nicht nur teale Geschehnisse geschildert, sondern $z$.B. auch fiktive Fallgeschichten (etwa im wirtschaftlichen oder juristischen Diskurs). Da diese fiktiven Elemente aber im Sinne der Praxisregulierung oder der Festlegung allgemeiner Strukturmerkmale mit Bezug auf die außersprachliche Wirklichkeit funktionalisiert werden, verstehen wir sie als Wirklichkeitserzählungen. Ferner finden sich (2) auf der Ebene des discours (der Ebene des Erzählens) häufig nicht-erzählende Passagen, etwa Zustandsbeschreibungen oder Argumentationen. Diese Textteile werden hier berücksichtigt, insofern sie funktional in die jeweilige Wirklichkeitserzählung eingebettet sind.

\section{Erzählen im sozialen Kontext}

Mehrfach wurde bereits die Position des 'Panfiktionalismus erwähnt, also jener Auffassung, derzufolge es keinen grundsätzlichen Unterschied gebe zwischen dem Wahrheitsanspruch eines fiktionalen bzw. eines faktualen Textes. Als ein Beispiel für viele sei die Literaturwissenschaftlerin Aleida Assmann zitiert, die die Differenz zwischen Fiktion und Realität als ein »verabschiedetes Paradigma« bezeichnet und behauptet: micht die Differenz, sondern die Indifferenz zwischen Fiktion und Realität ist das Datum, von dem heute ausgegangen werden muß«.10 Auch der Historiker Hayden White spricht bekanntlich von den angeblich unvermeidlichen "fictions of factual representations «. ${ }^{11}$ Diese Beobachtungen waren insofern wichtig, als sie auf die häufig vernachlässigte Bedeutung rhetorischer und konstruktiver Elemente des faktualen Erzählens aufmetksam machten. Sie haben inzwischen jedoch vielerorts zu dogmatischen Pauschalisierungen geführt, die eine angemessene Beschreibung der charakteristischen Leistungen von Wirklichkeitserzählungen verhindern.

Dabei gab es durchaus in verschiedenen Disziplinen Versuche, Formen und Funktionen faktualen Erzählens differenziert zu beschreiben. Die Texte, die wir hier unter den Begriff Wirklichkeitserzählung fassen, sind alles andere als homogen. Wie kann man sie systematisch erfassen und unter ihnen bestimmte Grundformen und -funktionen unterscheiden, um ihre jeweilige Rolle in der sozialen Konstruktion unserer Wirklichkeit zu beschreiben? Drei ältere Versuche, faktuales Erzählen zu typologisieren, seien kurz aufgeführt.

10 Aleida Assmann: „Fiktion als Differenz«, Poetica 21 (1989), S. 239-260, hicr: S. 240.

11 So der 'Titel eines Aufsatzes von Hayden White, abgedruckt in: Ders.: The Tropics of Discourse. Essays in Cultural Criticism, Baltimore 1978, S. 121-134. 
Sitrim Leben

Eine erste Typologie stammt aus der protestantischen Theologie und wurde zu Beginn des 20. Jahrhunderts in der sogenannten Religionsgeschichtlichen Schule von Hermann Gunkel entwickelt. Gunkel wollte die Bedeutung von Texten des Alten Testaments durch die Rekonstruktion ihrer ursprünglichen soziokulturellen Kontexte erklären: Im »alten Israel [ist] die Literatur ein Teil des Volkslebens und muß aus diesem begriffen werden ${ }^{12}$ Nun enthält das Alte Testament ganz unterschiedliche Gattungen: Psalmen, Sprichwörter, Legenden, Lieder, Hymnen, Prophezeiungen, Gebete, Listen, Annalen u.a. Gunkel meint, alle diese Gattungen seien jeweils in typischen Situationen der alltäglichen Lebenswelt des israelitischen Volkes gebraucht worden.

Wer also cine antike Gattung verstehen will, hat zunächst zu fragen, wo sie ihren Sity im Volksleben habe: den Rechtsspruch ж.B. zitiert der Richter vor Gericht zur Begründung der Entscheidung, und das Sicgeslied singen dic Mädchen beim Fïnzug des siegreichen Hecres. Sehr häufig wird auch die Gattung durch cinen Stand getragen, der über ihre Reinhcit wacht: so die ,Tora von den Priestern, die ,Weissagung، von den Propheten. ${ }^{13}$

Gunkel bestimmt Textgattungen also pragmatisch durch die Funktion, die sie in ihrem ursprünglichen "Sitz im Leben« erfüllen. In Gunkels Gattungsbegriff kommen somit drei Elemente zusammen: (a) Inhalt (sein bestimmter Schatz von Gedanken und Stimmungen«), (b) Form (»eine deutliche Formensprache, in der diese sich äußern«), (c) soziale Funktion (wein Sitz im Leben, aus dem Inhalt und Form erst verstanden werden können $).{ }^{14}$

\section{Einfache Formen}

Einen anderen Ansatz findet man im Konzept der Einfachen Formen von André Jolles. In seinem gleichnamigen Buch, das der morphologischen Literaturwissenschaft der 1920er Jahre entstammt, unterscheidet Jolles neun Einfache Formen, die er zwar mit gängigen Gattungsnamen bezeichnet, aber ganz neu bestimmt: Legende, Sage, Mythe, Rätsel, Spruch, Kasus, Memorabile, Märchen, Witz. ${ }^{15}$ Auch Jolles' Typologie bezieht lebensweltliche Kommunikationssituationen, abstrakte Erzählstrukturen und sprachlich-literarische Verfahren der Textgestaltung aufeinander. Jeder Einfachen Form ist eine lebensweltliche Standardsituation (Jolles: »Geistesbeschäftigung() zugeordnet. Die Einfache Form als solche ist als

12 Hermann Gunkel: „Die israelitische Literatur« [1906], in: Ders.: Hermann Gunkel zur israelitischen Literatur und Literaturgeschichte, hg. v. Rüdiger Liwak, Waltrop 2004, S. 1-60, hier. S. 3 $\mathrm{Vgl}$. Andreas Wagner: wGattung und sitz im Lebenc. Zur Bedeutung der formgeschichtlichen Arbcit Hermann Gunkels (1862-1932) für das Verstehen der sprachlichen Größe T'ext«, in: Susanne Michaelis/Doris Tophinke (Ig.): Texte - Konstitution, Lerarbeitung, Typike. München/Newcastle 1996, S. 117-129.

13 Gunkel ( $\mathrm{Anm} .12)$, S. 3.

14 Gunkel (Anm. 12), S. 57.

15 André Jolles: Einfache Formen. Legende - Sage - Mythe - Rätsel-Spruch - Kasus - Memorabile Märchen - Witz, Halle 1930. Von den neun Einfachen Formen sind swei, Rätsel und Spruch, keinc narrativen Gattungen. 
ein invariantes abstraktes Schema zu verstehen, das in unterschiedlicher Weise, je nach gegebenem kulturellen Rahmen, als faktual-pragmatische Textsorte realisiert wird ("gegenwärtige Form«), aber auch in ästhetisch-literarischer Gestalt ("Kunstform () oder auch abgewandelt oder hybridisiert (»bezogene Form(s) erscheinen kann. Jolles entwickelt damit eine kontextbezogene Typologie des Erzählens. Der Zugriff auf die Wirklichkeit der Lebenswelt mit Hilfe der Einfachen Formen ist dabei durchaus flexibel konzipiert: Die kommunikative Bedeutung eines Ereignisses (z.B. die Herrschaft Napoleons oder der Weltmeistertitel für die deutsche Fußballnationalmannschaft 1954), aber auch seine textliche Gestaltung können ganz verschieden ausfallen, je nachdem, ob es z.B. als Gründungsmythos (Sage), als Verklärung von Heiligen (Legender), als Wunscherfüllung (Märchen`), als Darstellung eines kasuistischen Normenkonfliktes (Kasusi) oder als prägnante historische Begebenheit (Memorabiler) ausgeformt wird. Die Einfachen Formen gestalten so in einem »mittleren Allgemeinheitsgrad« jeweils bestimmte »Subsinnwelten« (Hans Robert Jauß, mit Bezug auf William James und auf Alfred Schürz' und Thomas Luckmanns Strukturen der Lebenswelt). ${ }^{16}$ Literarische Texte berücksichtigen sie eher am Rande und zielen vor allem auf faktuale Erzählungen unter Berücksichtigung ihrer pragmatischen und soziokulturellen Kontexte. Diese Orientierung an Kontexten des Erzählens wurde in der späteren, formalistisch und strukturalistisch geprägten Narratologie vernachlässigt. Die Theorie der Einfachen Formen wurde besonders in der literaturwissenschaftlichen Mediävistik (in der germanistischen von Hugo Kuhn, in der romanistischen von Hans Robert Jauß) aufgenommen. In der Volkskunde wird der Begriff ebenfalls verwendet. Man bezeichnet hier mit Einfacher Form pauschal die Textsorten der Alltagskommunikation - der systematische Anspruch von Jolles' Theorie ist dabei verschwunden. Allerdings hat auch Jolles selbst diesen Anspruch nicht wirklich eingelöst. Obwohl er in seinem Buch vom »geschlossenen System unsrer Einfachen Formen « spricht, ${ }^{17}$ begründet er nirgendwo systematisch die unterstellte Vollständigkeit seiner neun Typen.

\section{Kommunikative Gattungen}

Der dritte und jüngste Versuch, Erzählgattungen systematisch über ihre soziale Funktion zu definieren, wurde in der verstehenden Soziologie der 1980er Jahre entwickelt: Thomas Luckmanns Konzept der sommunikativen Gattungen. Mit diesem Begriff bezeichnet Luckmann "gesellschaftlich vorgeprägte und mit mehr oder minder verbindlichen Gebrauchsanweisungen versehene Muster kommuni-

16 Hans Robert Jauß: mAlterität und Modernität der mittelalterlichen Literaturı, in: Ders: Alterität und Modernität der mittelalterlichen Literatur. Gesanmmelte Anfsätze 1956-76, München 1977, S. 9-48, hier: S. 39 u. 41. Vgl. Ulla Fix: $»$ Was ist aus André Jolles' ,Einfachen Formen heute geworden? Eine kulturanalytische und textlinguistische Betrachtung«, in: Volker Hertel (Hg.): Sprache und Kommunikation in Kulturkontext, Fs. Gotthard Lerchner, Frankfurt a.M. 1996, S. 105-120.

17 Jolles (Anm. 15), S. 172. 
kativen Handelns ${ }^{18}$ Die grundlegende Funktion kommunikativer Gattungen, so Luckmann, liege in der Sicherung sozialer Ordnung durch die Bereitstellung von sprachlichen Verhaltensmustern zur Bewältigung spezifischer kommunikativer Probleme. Kommunikative Gattungen bestehen aus einer verfestigten (allerdings historisch und kulturell spezifischen) Verbindung vorgeprägter Elemente, die in aktuellen Situationen variabel angewendet werden können. Zu diesen Elementen gehören neben einem bestimmten sprachlichen und außersprachlichen Repertoire von Ausdrucksmitteln und Stilebenen eine korrespondierende soziale Situation sowie der soziale Status der Kommunikationsteilnehmer und deren jeweilige Rolle im Vollzug der kommunikativen Gattung. Einzelne kommunikative Gattungen, die Luckmann und andere untersucht haben, sind das Gerücht, der Klatsch, die Predigt, die Alltagsgeschichte, die Beratung und die Konversionserzählung.

Die Ansätze von Gunkel, Jolles und Luckmann haben drei Dinge gemeinsam: (a) Sie ordnen den Bereich faktualen Erzählens mit Hilfe einer Gattungstypologie, (b) sie weisen den Gattungen jeweils bestimmte soziale Funktionen zu, und (c) sie leiten spezifische Textmerkmale dieser Gattungen aus ihrer jeweiligen sozialen Funktion ab. Alle drei Konzepte haben aber Schwierigkeiten, den Status der postulierten Typologie systematisch zu begründen. Gunkel bezieht sein Konzept vom Sitz im Leben ohnehin nur auf alttestamentarische Texte, ohne eine allgemeine Gattungstypologie faktualen Erzählens zu beabsichtigen. Jolles hingegen erhebt durchaus einen systematischen Anspruch, ohne aber die Vollständigkeit seiner neun Einfachen Formen zu rechtfertigen. Luckmann unterstellt ebenfalls, dass die kommunikativen Gattungen, die eine bestimmte Gesellschaft benutzt, ein geschlossenes System bilden. Aber auch er liefert zwar Untersuchungen zu einzelnen Gattungstypen, präsentiert aber keine umfassende oder gar systematisch abgeschlossene Übersicht existierender kommunikativer Gattungen.

\section{Felder und Leitdifferenzen}

Angesichts der Beobachtung, dass Wirklichkeitserzählungen in allen Bereichen sozialer Kommunikation eine wichtige Rolle spielen, stellt sich die Frage, wie man diese Bereiche voneinander abgrenzen kann. In den Gesellschaftswissenschaften konkurrieren verschiedene Beschreibungsversuche gesellschaftlicher Teilbereiche und ihrer Differenzierung, von denen die Konzepte Pierre Bourdieus und Niklas Luhmanns besonders wirkungsmächtig geworden sind.

18 Thomas Luckmann: »Der kommunikative Aufbau der sozialen Welt und die Sozialwissenschaften« [1995], in: Ders.: W'issen und Gesellschaft. Ausgewählte Aufsä̈že 1981-2002, Konstanz 2002, S. 157-181, hicr: S. 165; Ders.: )Grundformen der gesellschaftlichen Vermittlung des Wissens: Kommunikative Gattungen«, in: Fricdhclm Neidhardt u.a. (Hg.): Kultur und Gesellschaft, Opladen 1986, S. 191-211; Susanne Günthner: „Von Konstruktionen ‘u kommunikativen Gattungen. Die Relevanz sedimentierter Muster für die Ausführung kommunikativer Aufgaben«, Deutsche Sprache 34 (2006), S. 173-190. 
Für Pierre Bourdieu lässt sich Gesellschaft in verschiedene Felder aufteilen, die als relativ eigenständige und nach je eigenen Gesetzmäßigkeiten strukturierte Bereiche zu verstehen sind. Die Geschichte eines Feldes ist dabei ein Kampf um die Etablierung legitimer Wahrnehmungs- und Bewertungskategorien. ${ }^{19}$ Im Zuge der Autonomisierung des Feldes bilden sich die jeweils spezifischen Instanzen und Mechanismen zur Durchsetzung dieser Ansprüche heraus. Je autonomer schließlich das Feld ist, desto unabhängiger sind die Bewertungskategorien von denen anderer Felder. ${ }^{20}$ Über die spezifische Position der einzelnen Akteure im Feld entscheidet die Summe des für das spezifische Feld relevanten Kapitals. ${ }^{21}$

Auch bei Niklas Luhmann ist der Aspekt der gesellschaftlichen Differenzierung ein Kernpunkt der Theorie. Er entwirft eine Art Evolution gesellschaftlicher Differenzierungsformen: Auf eine erste Phase der segmentären Differeñierung (Gesellschaft spalte sich auf in zahlreiche gleiche Einheiten wie Familien, Stämme etc.) folge eine zweite Phase: die der stratifikatorischen Differenqierung. Hier teile sich Gesellschaft in ungleiche, hierarchisch organisierte Schichten auf, wobei die Kriterien, nach denen diese Differenzierung erfolge, letztlich sekundär seien. Ungeachtet der verschiedenen sozialen Positionen teilten alle Mitglieder der Gesellschaft die gleiche Grundsymbolik (nämlich eine im Wesentlichen religiös fundamentierte Seins- und Weltauslegung). Seit dem 16. Jahrhundert sei die Gesellschaft zunehmend komplex geworden und habe sich in einer dritten Phase in Teilsysteme ausdifferenziert, die nicht mehr über eine gemeinsame Grundsymbolik verfügten (funktionale Differeñierung) - diese Entwicklung schlage spätestens zur Mitte des 19. Jahthunderts durch. ${ }^{22}$ In dieser dritten Phase sei für die Differenzierung der jeweiligen Teilsysteme ihre gesellschaftliche Funktion entscheidend: Jedes Teilsystem habe eine exklusive gesellschaftliche Funktion, die die anderen Teilsysteme nicht übernehmen könnten. Jedes Teilsystem operiere mit Hilfe spezifischer Grundunterscheidungen, den binären Codes, denen sogenannte Leitdifferenzen zugrunde lägen.

Wir wollen Luhmanns Idee, verschiedene gesellschaftliche Teilbereiche anhand unterschiedlicher Leitdifferenzen zu unterscheiden, für eine Typologie faktualen Erzählens übernehmen. Für die in diesem Band vertretenen Felder wären folgende Leitdifferenzen anzusetzen: Rechtswissenschaft: legal/illegal; ${ }^{23}$ Medizin und Psychologie: gesund/krank bzw. erweiternd: lebensförderlich/lebenshinderlich; ${ }^{24} \mathrm{~W}$ issenschaft: wahr/unwahr; ${ }^{25} \mathrm{Wirtschaft:}$ zahlen/nicht-zahlen; ${ }^{26}$ Moral:

19 Jierre Bourdicu: Die Regeln der Kunst. Genese und Stmktur des literarischen Feldes, J'rankfurt a.M. 1999, S. 253.

20 Vgl. Bourdieu (Anm. 19), S. $116 \mathrm{ff}$

21 Picrrc Bourdicu: Die feinen Unterschiede. Kritik der gesellschaftlichen Urteilskraft, Frankfurt a.M. 1987, S. 194f. Vgl. zum Konzept der verschiedenen Kapitalsorten auch: Pierre Bourdicu: „Ökonomisches Kapital, kulturelles Kapital, soziales Kapital«, in: Reinhard Kreckel (Hg.): Soziale Ungleichbeiten, Göttingen 1983, S. 183-198.

22 Vgl. hicrzu cinführend: Niklas Luhmann: wGesellschaftliche Struktur und semantische Tradition«, in: Ders.: Gesellschaftsstruktur mo Semantik. Studien qur Wissenssoziologie der modernen Gesellschaft, Bd. 1, Frankfurt a.M. 1980, S. 9-71.

23 Niklas Juhlmann: Das Recht der Gesellschaft, Frankfurt a.M. 1995.

24 Jost Bauch: Gesmndheit als sozialer Code. Von der Vergesellschafiung des Gesundheitswesens zur Me- 
gut/böse bzw. im Bezug auf Handlungen: richtig/falsch;27 Journalismus: aktuell/ nicht-aktuell bzw. informativ/nicht-informativ; ${ }^{28}$ Religion: dem Heil förderlich/ dem Heil nicht förderlich;29 Politik: Regierung/Opposition bzw. Amtsmacht/ ohne Amtsmacht. ${ }^{30}$ Das Konzept dieses Bandes ordnet den Bereich der Wirklichkeitserzählungen systematisch anhand dieser spezifischen Leitdifferenzen, die das Erzählen in den verschiedenen Feldern sozialer Kommunikation jeweils bestimmen. Ergänzt werden müssen diese Teilsysteme differenzierenden Leitdifferenzen durch zwei grundsätzliche Unterscheidungen, die quer zu den gesellschaftlichen Teilsystemen liegen, weil sie letztlich für alle Kommunikationsteilnehmer gelten, unabhängig davon, in welchem Teilsystem sie agieren: $i c h / d u$ (Typ der Wirklichkeitserzählung: Ego-Erzählungen) sowie wir/ihr (Typ der Wirklichkeitserzählung: Kollektiverzählungen).

Luhmanns Ansatz ermöglicht einen differenzierten Blick auf die verschiedenen gesellschaftlichen Felder, in denen Wirklichkeitserzählungen eine Rolle spielen. Die Orientierung an spezifischen Leitdifferenzen fokussiert die Betrachtung der jeweils kursierenden Wirklichkeitserzählungen auf die Eigenheiten der Kommunikation innerhalb der jeweiligen Teilsysteme: So kreist die Kommunikation im juristischen Diskurs vor allem um die Frage, ob etwas rechtmäßig sei oder nicht, im wirtschaftlichen Diskurs um die Frage, ob sich eine Investition lohne oder nicht usw.

Als analytisches Instrumentarium ist diese Differenzierung hilfreich. Als Beschreibungsmodell für moderne Gesellschaften scheint Luhmanns Ansatz indes zu starr, schließt die zweiwertige Codienung der Teilsysteme doch alles aus, was nicht innerhalb des durch die Leitdifferenz markierten Raumes Platz hat. Hier bietet wiederum Bourdieus Feldtheorie mehr Offenheit. Denn für Bourdieu existieren zwischen den einzelnen sozialen Feldern (die in ihrer Funktion durchaus Luhmanns Teilsystemen vergleichbar sind) strukturale und funktionelle Homologien, die dazu führen, dass eine besonders einflussreiche Stellung in einem Feld die Einflussmöglichkeiten innerhalb eines anderen Feldes vergrößert. ${ }^{31}$ So kann z.B. ein Politiker Entscheidungen treffen oder beeinflussen, die das Feld der Wirtschaft betreffen usw. Deshalb scheint es sinnvoll, das Konzept der gesellschaftlichen Felder mit der Idee der Leitdifferenzen zu verbinden.

dikalisientng der Gesellschaff, Weinheim/München 1996, hier: S. 12ff.

25 Niklas Luhmann: Die Wissenschaft der Gesellschaft, Frankfurt a.M. 1992.

26 Niklas Luhmann: Die Wirtschaft der Gesellschaft, Frankfurt a.M. 1994.

27 Niklas Luhmann: Die Moral der Gesellschaft, hg. v. Detlef Horster, Frank furt a.M. 2008.

28 Maja Malik: Journalismusjournalismus. Funktion, Strukturen und Strategien der jounalistischen Selbstthematisiering, Wiesbaden 2004, S. 35-56.

29 Niklas Iuhmann: Die Religion der Gesellschaft, hg. v. André Kicserling, Frankfurt a.M. 2002

30 Niklas Juhmann: Die Politik der Gesellschaft, hg. v. André Kiescrling, Frankfurt a.M. 2002.

31 Bourdicu (.Inm. 19), S. 291. 


\section{Terminologie und Aufbau der Beiträge}

Der vorliegende Band möchte einen systematisierten interdisziplinären Zugriff auf das breite Forschungsfeld der Wirklichkeitserzählungen vorstellen, Perspektiven eröffnen und $z u$ weiterer Beschäftigung mit faktualem Erzählen anregen dass er nicht den Anspruch erhebt, das Thema erschöpfend zu behandeln, versteht sich von selbst. Interdisziplinarität birgt dabei stets Chancen, ist aber auch riskant. Dieser Band enthält Beiträge von Autorinnen und Autoren verschiedener wissenschaftlicher Disziplinen über unterschiedliche soziale Felder, in denen Erzählungen Wissen und Verhaltensweisen vermitteln. Da diese Felder divers und die für sie jeweils zuständigen wissenschaftlichen Disziplinen eigene Begriffsinventare und Analysemethoden herausgebildet haben, könnte die interdisziplinäre Anlage unseres Bandes im schlechtesten Fall einen weiteren Beleg dafür liefern, dass eine echte Verständigung über die Grenzen unterschiedlicher Gegenstandsbereiche und Disziplinen hinweg oft mehr beschworen als eingelöst wird. Unser Ziel war es jedenfalls, den Eigenheiten des Erzählens in den verschiedenen Feldern angemessen Rechnung zu tragen, ohne deshalb den Anspruch auf eine übergreifende Systematik und vergleichende Zusammenschau aufzugeben.

Die Beiträge sollen diesen Anspruch über zwei Wege einlösen. Zum einen verwenden sie (wo es geraten scheint) ein in der philologischen Erzähltextanalyse inzwischen weithin gebräuchliches erzähltheoretisches Instrumentarium, das vor allem von Gérard Genette geprägt wurde. Genette unterscheidet im Hinblick auf die Art und Weise des Erzählens drei Aspekte: ${ }^{32}$ (a) Zeit (Verhältnis zwischen Zeit der Erzählung und Zeit des Geschehens), (b) Modus (Mittelbarkeit und Perspektivierung des Erzählten) und (c) Stimmze (Akt des Erzählens, Verhältnis von Erzähler, Erzähltem und Leser). Im Hinblick auf die Besonderheiten von Wirklichkeitserzählungen scheint vor allem die Kategorie des Modus relevant und hier (erstens) die Frage nach der Distanz (wie mittelbar wird das Erzählte präsentiert?) sowie (zweitens) die Frage nach der Fokalisierung (aus welcher Sicht wird erzählt?).

Zum anderen liegt allen Beiträgen ein einheitlicher Aufbau zugrunde: Sie beginnen mit einer Kurzbestimmung des Wirklichkeitsfeldes und gehen dabei auch auf die dominante Leitdifferenz ein, die dieses Feld charakterisiert. Daran schließt ein systematischer Überblick über die spezifischen Formen und Funktionen des Erzählens in dem diskutierten Wirklichkeitsfeld an. Es folgen exemplarische Einzelfalldarstellungen, bevor in einem forschungsgeschichtlichen Uberblick aktuelle Probleme und Desiderate der Forschung angerissen werden. Die Beiträge schlieBen mit einer kommentierten Auswahlbibliographie zur weiterführenden Lektüre.

32 Vgl. zum Folgenden: Gérard Genette: Die Eräählang, München 1994, und Martínęz/ Scheffel (Anm. 4), Kap. II.1-3 (S. 27-89). 
Christian Klein/Matías Martínez (Hrsg.)

\section{Wirklichkeitserzählungen}

Felder, Formen und Funktionen

nicht-literarischen Erzählens

Verlag J. B. Metzler Stuttgart · Weimar 